\title{
Temperature and Humidity Data Acquisition System
}

\author{
Shao Jun ${ }^{1, a}$, Qian Ting ${ }^{2, b}$ \\ ${ }^{1}$ China Tobacco Zhejiang Industrial Co.,Ltd., Hangzhou 310024, China; \\ ${ }^{2}$ China Tobacco Zhejiang Industrial Co.,Ltd., Hangzhou 310024, China. \\ ashaojun@tobacco.com, b122601670@qq.com
}

\begin{abstract}
Keywords: MODBUS protocol; temperature and humidity; data acquisition; RTU mode
\end{abstract}
\begin{abstract}
Recently, as the increasing requirement on the stability, reliability and accuracy of environmental temperature and humidity data in the Tobacco Industry, a set of correcting ways of temperature humidity sensors has been fulfilled and a Data Acquisition System based on the MODBUS protocol has been designed and performed with the equipment of a temperature and humidity environment experimental case. The system includes data acquisition module,data processing module and data display and memory module, which using the SQL Server as the background data server. Moreover, the system communication applies the MODBUS RTU mode, resulting in the successful realization of temperature and humidity data acquisition, display, storage and inquires functions.
\end{abstract}

\section{Introduction}

As the development of manufacture and technology in Tobacco industry, we focus more on the influence from the environment of the factory, especially during the primary process and packaging stage. It is very important to control the moisture and temperature, because the temperature and humidity in the workshop can affect the storage of the tobacco leaves and quality of rolled tobacco, which are closely related to the final quality of the cigarette. It raised a very high standard for the equipment to measure environmental temperature and humidity, to help to control the temperature and humidity within proper range, and to provide a comfortable working condition for the operators.

Nowadays the measurement of temperature and humidity calibration data mainly depends on human work, which has some issues like low accuracy and not in real time. To increase the accuracy of calibration of temperature and humidity sensor, and high reliability of data capture, it is crucial to use an efficient and reliable data acquisition system. This article proposes a new data acquisition system $^{[1]}$ in real-time, based MODBUS protocol and Delphi, SQL ${ }^{[2]}$ database as programming platform.

\section{System Structure}

The temperature and humidity calibration system software in this article was partially developed by the writer, and it is strong at portability and has a good potential of further development. The system mainly includes: humidity environment experimental case, protimeter hygromaster, data collector and monitoring computer system. The designed procedure of this system: Placing the temperature and humidity sensors evenly in the humidity environment experimental case, setting the target value of temperature and humidity, connecting the sensors and data acquisition module, the testing result is transmitted to the data collector via electric signal, and the test results are recorded and transferred to the monitoring computer by MODBUS protocol, the computer reads the data in the collector periodically according to the time interval set in the computer before, and displays on the screen in real time in the form of number and plotted curves, user can search and export the data to further analysis and calibrate the protimeter hygromaster sensors. 
The entire data acquisition module includes: humidity environment experimental case, temperature and humidity sensors, data collector and monitoring computer system (data acquisition module, data processing module and display storage module).

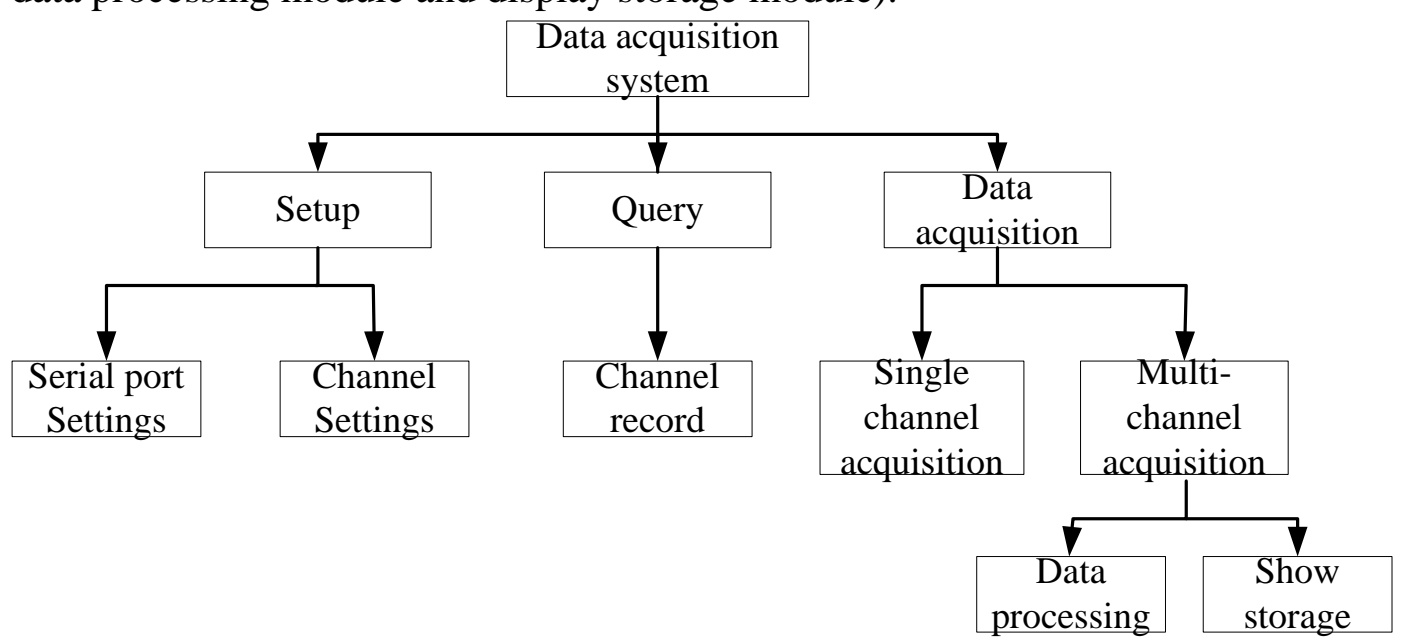

Fig.1 Software structure chart

The design of the system is implemented as: setting up the humidity environment experimental case to target temperature and humidity value, the sensors pass the temperature and humidity data to data collector using voltage and current signals, the data collector records the data in real time, monitoring computer reads the data in the collector given the time interval via MODBUS RTU protocol by RS-232C, after the format conversion processing, the computer displays the testing result and plotted charts, the operator calibrate the sensor according to the test result, to ensure the accuracy of the sensors.

\section{Hardware Structure}

The humidity environment experimental case is prepared on the basis of the test requirement, which can create an environment with the temperature ranging from $-40^{\circ} \mathrm{C} \sim 180^{\circ} \mathrm{C}$ and humidity ranging from 10\% 98\%, and the relative humidity is controlled by Michell Dew Point meter. The dew point meter is treated as a standard equipment and temperature and humidity sensor which needs to be calibrated transmit the signal to the data collector, which can use 18 channels (i.e. 9 temperature and humidity sensors) maximally at the same time. Finally the monitoring computer reads the real time data according to the set period via MODBUS protocol.

\section{Software Design}

\section{MODBUS Introduction}

MODBUS protocol is universal program language applied on electric controller. Through this control, controller can communicate with each other or other devices via network (such as Ethernet). It has become a popular industrial standard. Standard MODBUS port uses RS-232RC[3] compatible serial port, which defines interface pins, cable, signal level, baud rate and parity check.Standard MODBUS protocol[4] has two transmission mode: ASCII mode and RTU mode. In ASCII mode, each 8bit in the message are treated as two ASCII characters, with LRC checking. The advantage of this mode is there is no error when the interval between sending character is as short as 1 second. In RTU mode, the protocol uses bit transfer and CRC check. The advantage of this mode is that it can deliver more data compared with ASCII mode at the same baud rate.

\section{MODBUS RTU communication protocol implementation}

According to the definition of the data structure in MODBUS RTU protocol, hexadecimal system is used in the data format, and 32-bit floating point system is used in the data transfer. When the master device transmit command communication to the slave device, the slave matches the address code receives the command and reads corresponding message according to the function code. If the 
CRC check is correct, the slave will execute the task and send the result back to the master, including the address code, function code and result as well as the CRC check code. If CRC check fails, no message will be returned.

As the format of floating point number, the MODBUS protocol in this system uses single-precision floating point number which is slightly different from IEEE-754. Table 1 shows the format, and S stands for symbol bits, E stands for exponent and $\mathrm{M}$ stands for the ending bit.

Table 1 Single-precision floating point number format

\begin{tabular}{ccc}
\hline Standard & Low bits & High bits \\
\hline \multirow{2}{*}{ IEEE-754 } & SEEEEEEE & MMMMMMMM \\
& EMMMMMMM & MMMMMMMM \\
\multirow{2}{*}{ MODBUS } & MMMMMMMM & SEEEEEEE \\
& MMMMMMMM & MMMMMMMM \\
\hline
\end{tabular}

\section{Function module implementation}

\section{Data acquisition serial module}

Based on the output format of serial communication module in MODBUS protocol, we use the third party widget TMS.Async32 serial communication module[5] in Delphi programming tool, according to the format of MODBUS protocol, and write the data acquisition interface program.

\section{Single-precision floating point number processing module}

In the data acquisition module, it is crucial to keep the data processing in real time and accurate to ensure the stability of the entire collecting system, especially the conversion format between Single-precision floating point number and MODBUS protocol data structure, which uses hexadecimal system according to the format of Single-precision floating point number and IEEE-754 standard.

(1) Master sending command: Master sends out data command in real time, according to pre-set sampling period. CRC16 check is added at the end of the message before the transmission, and the master waits for the reply from slave at the same time.

(2) Master receiving command: Master receives the data first, then use CRC16 check the correctness of the replied message, then convert the character string to hexadecimal. Then the data is converted to Binary and then to decimal with integer and decimal part finally[6]. The transfer error message will be displayed if there is any problem during the CRC16 checking.

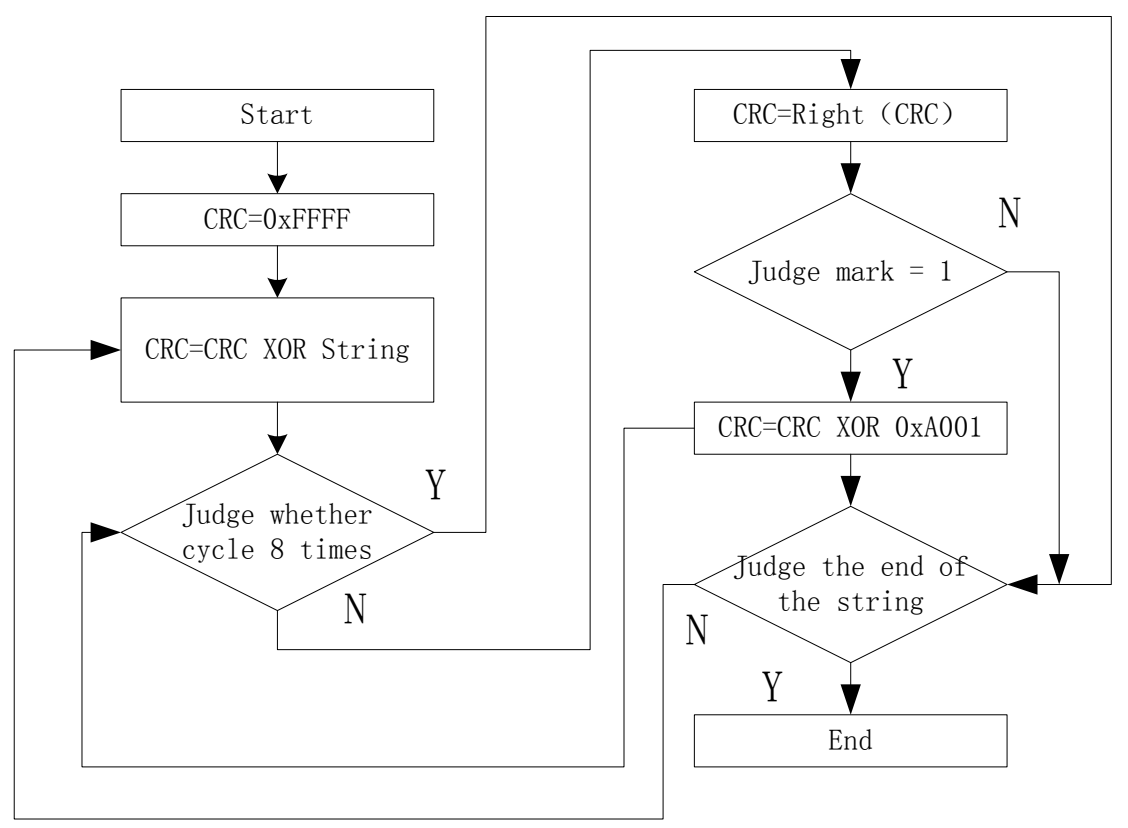

Display and storage module

Fig. 2 CRC checking flow chart 
After opening the serial port under control menu in the data acquisition window[7], user can record data in each channel within sampling period. This system can connect to 18 channels (9 temperature and humidity meters) maximally, and 18 channels are divided into 3 groups, which show three temperature and humidity value respectively.

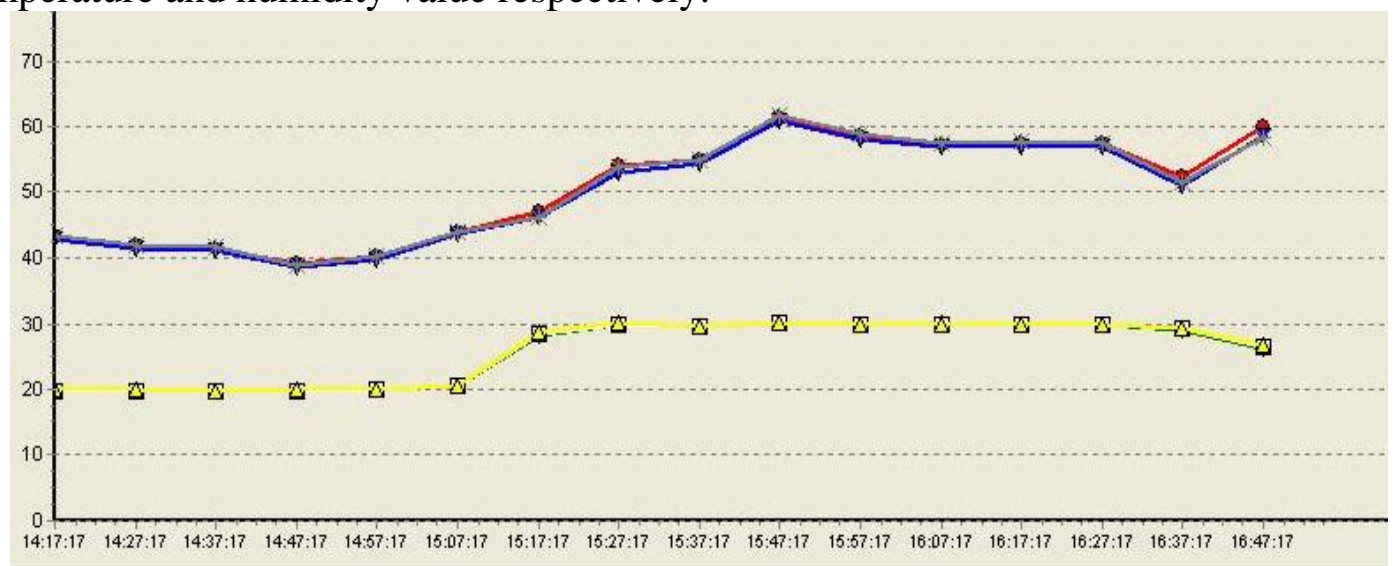

Fig. 3 temperature and humidity value chart

Temperature and humidity Data Acquisition System implements two-point calibration

According to the temperature and humidity calibration system, we calibrate EE22 temperature and humidity meter using two-point calibration, and linearize the curve between two points so the best measurement can be achieved. Since the ambient temperature is control around $20-30^{\circ} \mathrm{C}$, and $\mathrm{RH}$ is controlled between $40 \sim 80 \%$, we use $20^{\circ} \mathrm{C}$ as the low temperature point and $40 \%$ as the low humidity point; and high calibration point: $30{ }^{\circ} \mathrm{C}$ for temperature and $80 \%$ for $\mathrm{RH}$. The accuracy of the temperature and humidity meter is $\pm 0.5^{\circ} \mathrm{C}$ for temperature and $\pm 3.0 \%$ for relative humidity.

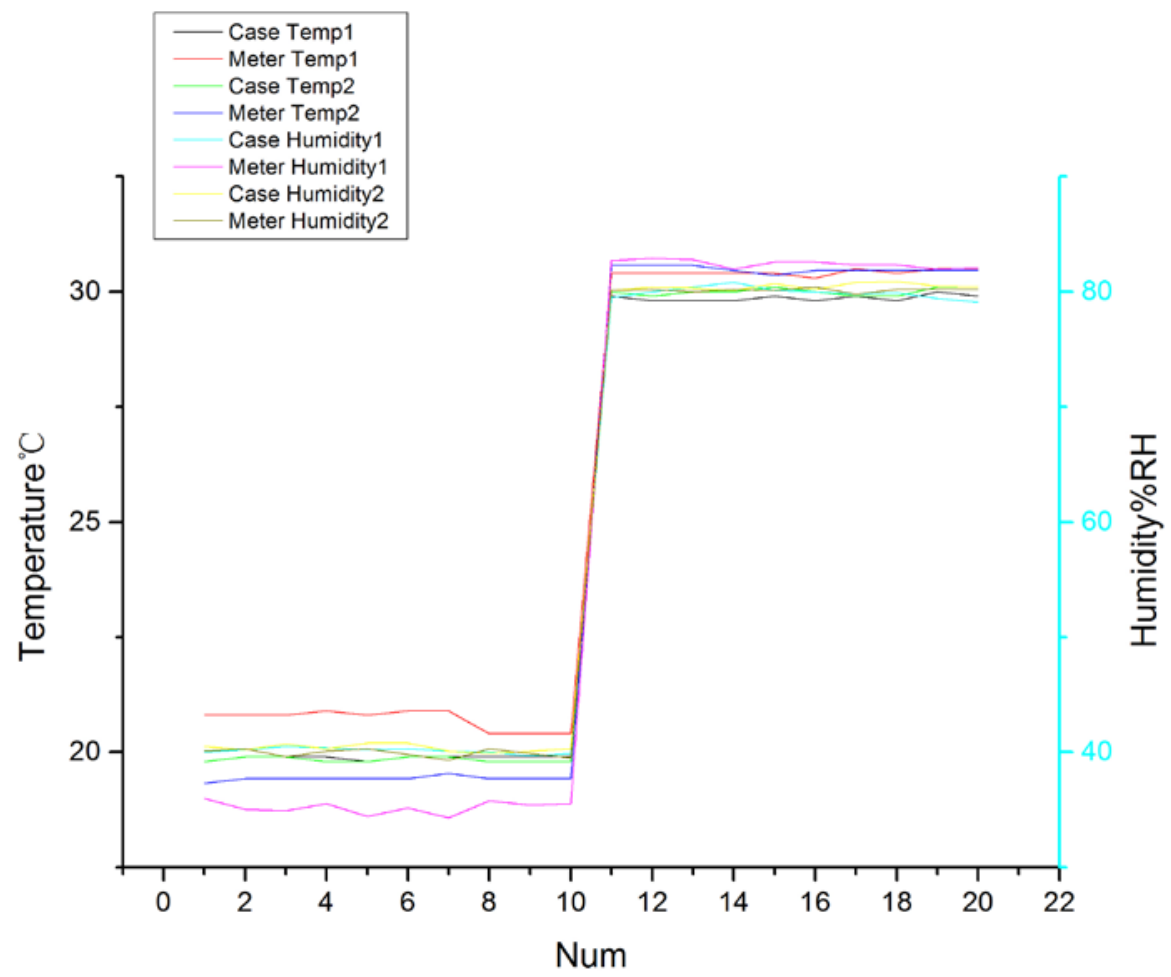

Fig. 4 using two-point calibrate temperature and humidity value chart

\section{Summary}

This solution of temperature and humidity collection system uses flexible serial communication to achieve a multi-point temperature and humidity collection in real time. This collecting system uses MODBUS protocol to implement the communication between the master and slave devices[8], with 18 channels. It uses a mature specification and communication protocol, so the error rate is low and 
reliability is high, and in real time and the integrity is ensured. As a result, the calibration of temperature and humidity sensor is accurate and reliable so the request of temperature and humidity control during cigarette manufacture in the corporation can be well fulfilled.

\section{References}

[1] Sheng Linhui, Modbus RTU serial communication application in industrial automation systems, Control and Instruments In Chemical Industry, 2014,41(2):207-211.

[2] Liu ZhiYong, SQL Server 2005 bible, Beijing: electronic industry press, 2007.

[3] Yang Yanhua, Zhou Yonglu. Design and Implementation of Protocol Converter Between General Serial Port and Modbus RTU, Computer Measurement and Control, 2014,22(2):604-606.

[4] Rev. J Modicon, MODBUS Protocol Reference Guide, MODICON INC., Industrial Automation System, June 1996.

[5] LiangShui, Li Fangchao, Shai KuiChun, Delphi development techniques, Beijing: publishing house of people's posts and telecommunications, 2007.

[6] Bao Lei, Ma Hong-fei, Servo-motor control system based on the single-chip microcomputer applied Modbus RTU communication protocol, Manufacturing Automation, 2014,36(3):48-51.

[7] Feng Licheng, About real-time data acquisition system optimization design in the LabVIEW technology, Electronics World, 2014,18:3-3.

[8] Jia Peigang, The design of multi-channel data real-time processing system based on LabVIEW, Manufacturing Automation,2014,36(17):128-131. 